\title{
High-dose IgG therapy mitigates bile duct-targeted inflammation and obstruction in a mouse model of biliary atresia
}

\author{
Erika K. Fenner ${ }^{1,2}$, Juri Boguniewicz', Rebecca M. Tucker ${ }^{1}$, Ronald J. Sokol ${ }^{2}$ and Cara L. Mack ${ }^{1,2}$
}

BACKGROUND: A proposed etiology of biliary atresia (BA) entails a virus-induced, progressive immune-mediated injury of the biliary system. Intravenous Ig (IVIg) has demonstrated clinical benefit in several inflammatory diseases. The aim of this study was to determine the therapeutic effects of high-dose IgG treatment in the rhesus rotavirus (RRV)-induced mouse model of BA.

METHODS: Newborn mice were infected with RRV, and jaundiced mice were given high-dose IgG or albumin control. Survival, histology, direct bilirubin, liver immune cell subsets, and cytokine production were analyzed.

RESULTS: There was no difference in overall survival between RRV-infected groups, however high-dose IgG resulted in decreased bilirubin, bile duct inflammation, and increased extrahepatic bile duct patency. High-dose IgG decreased vascular cell adhesion molecule-1, resulting in limited migration of immune cells to portal tracts. High-dose IgG significantly decreased $\mathrm{CD} 4^{+} \mathrm{T}$ cell production of interleukin (IL)-2, interferon (IFN)- $\gamma$, and tumor necrosis factor (TNF)- $a$ and $C D 8^{+}$ $T$ cell production of IFN- $\gamma$, as well as increased levels of regulatory $T$ cells.

CONCLUSION: High-dose lgG therapy in murine BA dramatically decreased Th1 cell-mediated inflammation and biliary obstruction. This study lends support for consideration of IVlg clinical trials in infants with $B A$, to diminish the progressive intrahepatic bile duct injury.

B iliary atresia (BA) is a progressive, inflammatory disease of the bile ducts resulting in neonatal cholestasis and characterized by fibrosis and obliteration of the extrahepatic and intrahepatic bile ducts (1). Despite surgical intervention with the Kasai portoenterostomy, the intrahepatic bile duct injury progresses, leading to biliary cirrhosis in the majority of children. The 10-y survival with the native liver ranges from $\sim 20$ $50 \%$ (2), and BA remains the leading indication for pediatric liver transplantation (3). The etiology of BA is unclear and multiple theories include developmental defects, virus infections, and immune dysregulation (4). A leading hypothesis is that a perinatal cholangiopathic virus infection initiates a chronic inflammatory response targeting bile duct epithelia, resulting in bile duct injury and fibrosis $(5,6)$.

An established mouse model of BA has been instrumental in elucidating the mechanisms of this aberrant immune response. Newborn mice infected with rhesus rotavirus (RRV) develop progressive inflammation and obstruction of the extrahepatic bile duct that recapitulates the early inflammatory damage seen in BA patients $(7,8)$. The inflammatory response is characterized by a Th1 cytokine milieu (9) with a subset of T cells showing autoreactivity to bile duct epithelial proteins $(10,11)$. In addition, increased levels of $\alpha$-enolase autoantibodies, common in other autoimmune hepatic and biliary diseases (12), have been detected in BA (13). The autoimmune responses may persist due to recently described deficits in regulatory $\mathrm{T}$ cells (Tregs) in both mouse and human BA (14-16).

We sought to determine if the inflammatory bile duct injury in the BA mice could be mitigated with high-dose polyclonal IgG treatment. In humans, intravenous Ig (IVIg) has been used to treat primary immunodeficiencies, and clinical benefit has been demonstrated in several autoimmune and inflammatory diseases (17-19). In mouse models of human disease such as immune thrombocytopenia (20), myasthenia gravis (21), viral myocarditis, (22) and experimental multiple sclerosis (23), human polyclonal IgG has been shown to have therapeutic benefit. Numerous mechanisms for the anti-inflammatory action of IVIg have been proposed, including interfering with the cytokine network, neutralizing autoantibodies, modulating the effector functions of $\mathrm{T}$ and $\mathrm{B}$ cells, and enhancing Tregs $(17,20,24)$. These mechanisms are not solitary and likely act synergistically.

The objectives of this study were to determine if high-dose IgG therapy in the mouse model of BA diminishes bile duct injury and to determine the possible mechanisms by which high-dose IgG modulates the hepatic immune response. Evaluating the potential therapeutic benefit of $\operatorname{IgG}$ is of clinical

The first two authors contributed equally to this work.

'Division of Allergy and Clinical Immunology, Department of Medicine, University of Colorado School of Medicine, Aurora, Colorado; ${ }^{2}$ Department of Pediatrics, Section of Pediatric Gastroenterology, Hepatology, and Nutrition, University of Colorado School of Medicine and the Digestive Health Institute, Children's Hospital Colorado, Aurora, Colorado. Correspondence: Erika K. Fenner (erika.fenner@ucdenver.edu) 
importance, given the paucity of therapeutic options currently available for patients with BA.

\section{RESULTS}

\section{High-Dose IgG Did Not Affect Overall Survival or Stunting of} Growth in RRV-Infected Mice

RRV-infected mice were observed for jaundice and acholic stools in the first week of life. In week $1, \sim 15 \%$ of mice died prior to day 7, 70\% became jaundice, and $15 \%$ were healthy. All jaundiced mice were treated with intraperitoneal injections of high-dose IgG (RRV-IgG, $n=56$ ), albumin control (RRV-alb, $n=47$ ), or neither (BA-RRV, $n=50$ ) on day 7 of life and every 2-3 d thereafter for six doses. Treatment administration was initiated on day 7 of life in mice that were deemed sick (jaundiced), as this was most clinically relevant to the human disease. There was no significant difference in overall survival and all mice expired by day of life 21 (Kaplan-Meier analysis, $P>$ 0.05) (Figure 1a). In similar fashion, the RRV-infected mice had diminished growth in weight, that did not improve in the IgG-treated mice (Figure 1b). To determine if viral clearance was affected by high-dose IgG therapy, infectious virus plaque assays were performed. A similar pattern of viral clearance was observed between RRV-alb and RRV-IgG groups, suggesting that the degree of virus infectivity did not play a role in survival outcomes (Supplementary Figure S1 online).

\section{High-Dose IgG Therapy Ameliorated Bile Duct Injury and Obstruction}

Serum direct bilirubin levels were used as a marker of bile duct injury and obstruction (Figure 2a). Whereas Hank's balanced salt solution (BSS) control mice had virtually undetectable levels of direct bilirubin at day of life 14, BA-RRV and RRV-albumin groups had markedly elevated levels of direct bilirubin, indicating significant bile duct injury $(\mathrm{mg} / \mathrm{dl}$ : BSS $0.19 \pm 0.1$; BA-RRV 11.02 \pm 0.9 ; RRV-alb 9.78 $\pm 1.0 ; P<0.0001$ ). In contrast, RRV-IgG mice had significantly lower levels of serum direct bilirubin $(4.75 \pm 0.73 \mathrm{mg} / \mathrm{dl})$ compared to both BA-RRV and RRV-alb groups $(P=0.0002)$.

Liver and extrahepatic bile duct histology was examined at $14 \mathrm{~d}$ of life to visualize the extent of bile duct inflammation and obstruction (Figure $2 b$ ). Portal tracts from BA-RRV and RRValb groups showed considerable portal tract inflammatory infiltration surrounding the intrahepatic bile ducts compared to minimal periductal immune cells in the BSS control or RRV-IgG groups. The extrahepatic bile duct histology showed a similar profile, with the BA-RRV and RRV-albumin groups demonstrating marked inflammation and luminal obstruction, compared to minimal inflammation and lack of bile duct occlusion in $100 \%$ of BSS and $70 \%$ of RRV-IgG mice.

\section{High-Dose IgG Therapy Decreased T Cell and Macrophage Portal Tract Infiltration by Means of VCAM-1 Inhibition}

To better quantify differences in the immunophenotype of liver immune cells in each group, flow cytometry was utilized. Liver immune cells were identified based on CD45 (hematopoietic) cell surface expression. At $10 \mathrm{~d}$ of life, the total liver immune cell
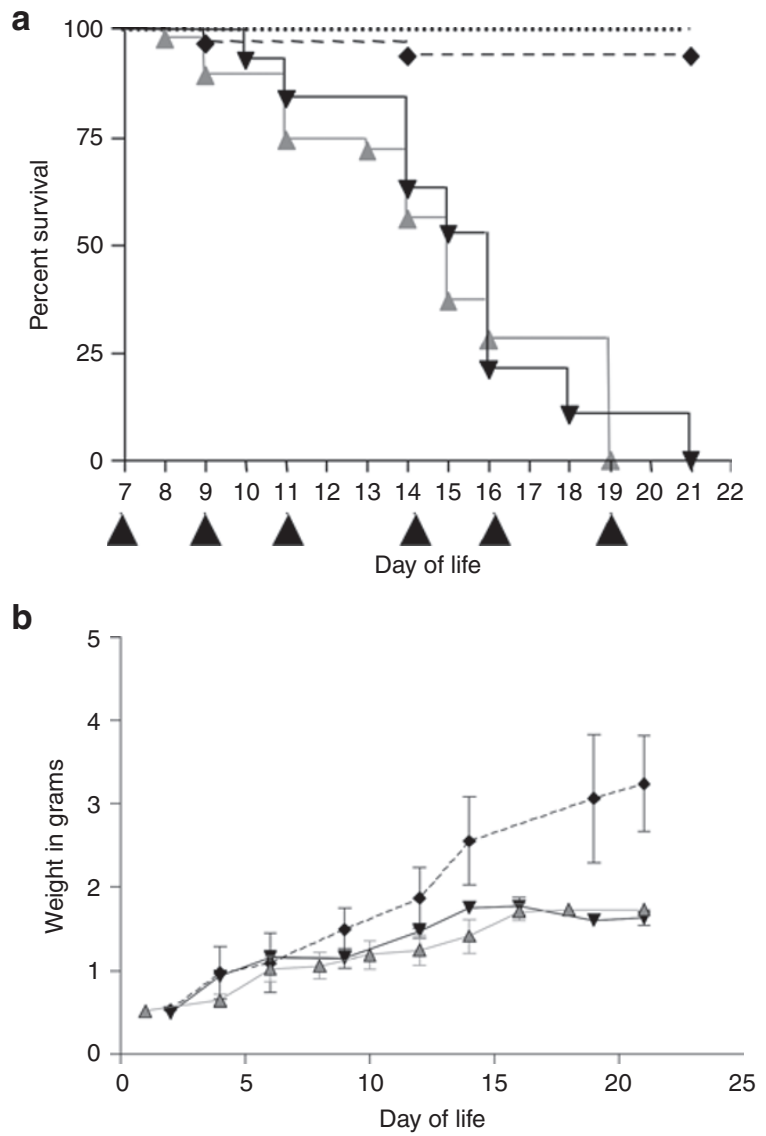

Figure 1. Survival. Newborn mice received BSS or RRV. On day 7 of life, RRV-infected, jaundiced mice received albumin (RRV-alb, (gray triangles) $n=47$ ) or lgG (RRV-lgG, (downward black triangles) $n=56$ ). Additional control groups included RRV-infected mice (BA-RRV, $n=50$ ) (mirrored RRV-alb and not shown), BSS-injected mice (BSS, (diamond symbol), $n=66$ ) and BSS-injected mice that received IgG (BSS-IgG, (dashed line) $n=17$ ). (a) Survival. Upward black triangles along the $x$-axis denote timing of albumin or lgG injections. There was no significant difference in survival in the RRV-infected groups (Kaplan-Meier; log rank test, $P>0.05$ ). (b) Weight. Total body weight was measured every 2-3 d in BSS, RRV-alb, and RRV-IgG groups. The RRV-infected mice had delayed growth compared to BSS, beginning at approximately day 10 of life. BSS, balanced salt solution; RRV, rhesus rotavirus.

profile (CD4 ${ }^{+} \mathrm{T}$ cells, $\mathrm{CD} 8^{+} \mathrm{T}$ cells, $\mathrm{CD} 11 \mathrm{~b}^{+}$macrophages) in all RRV-infected groups was similar, and all immune cell subsets were increased compared to the BSS control group (Figure 3a,b). The most significant increases were found in the macrophage population $\left(\% \mathrm{CD}_{11 \mathrm{~b}}+\right.$ : BSS $23.20 \pm 1.94 \%$, BA-RRV $43.38 \pm 3.77 \%, P=$ 0.0029 ; absolute number CD11 $\mathrm{b}^{+}$: BSS $1.74 \pm 0.11 \times 10^{6}$ cells/liver, BA-RRV $\left.4.04 \pm 0.22 \times 10^{6}, P=0.0015\right)$. There were no significant differences in surface markers for B cells and NK cells among any of the groups (data not shown). These data suggested that the IgG therapy did not change the overall relative abundance of the immune cell subsets within the entire liver.

In order to address the question of localization of the inflammatory cells within the liver, fluorescent immunohistochemistry was employed. Clusters of $\mathrm{CD} 4^{+}$and $\mathrm{CD} 8^{+} \mathrm{T}$ cells and $\mathrm{CD}_{11 \mathrm{~b}^{+}}$macrophages were expanded within the portal tracts of BA-RRV and RRV-alb mice but not in BSS controls or RRV-IgG 


\section{Articles | Fenneret al.}

a

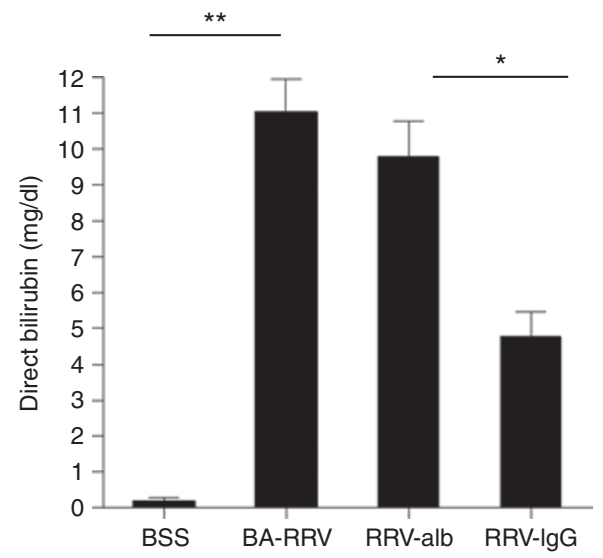

b
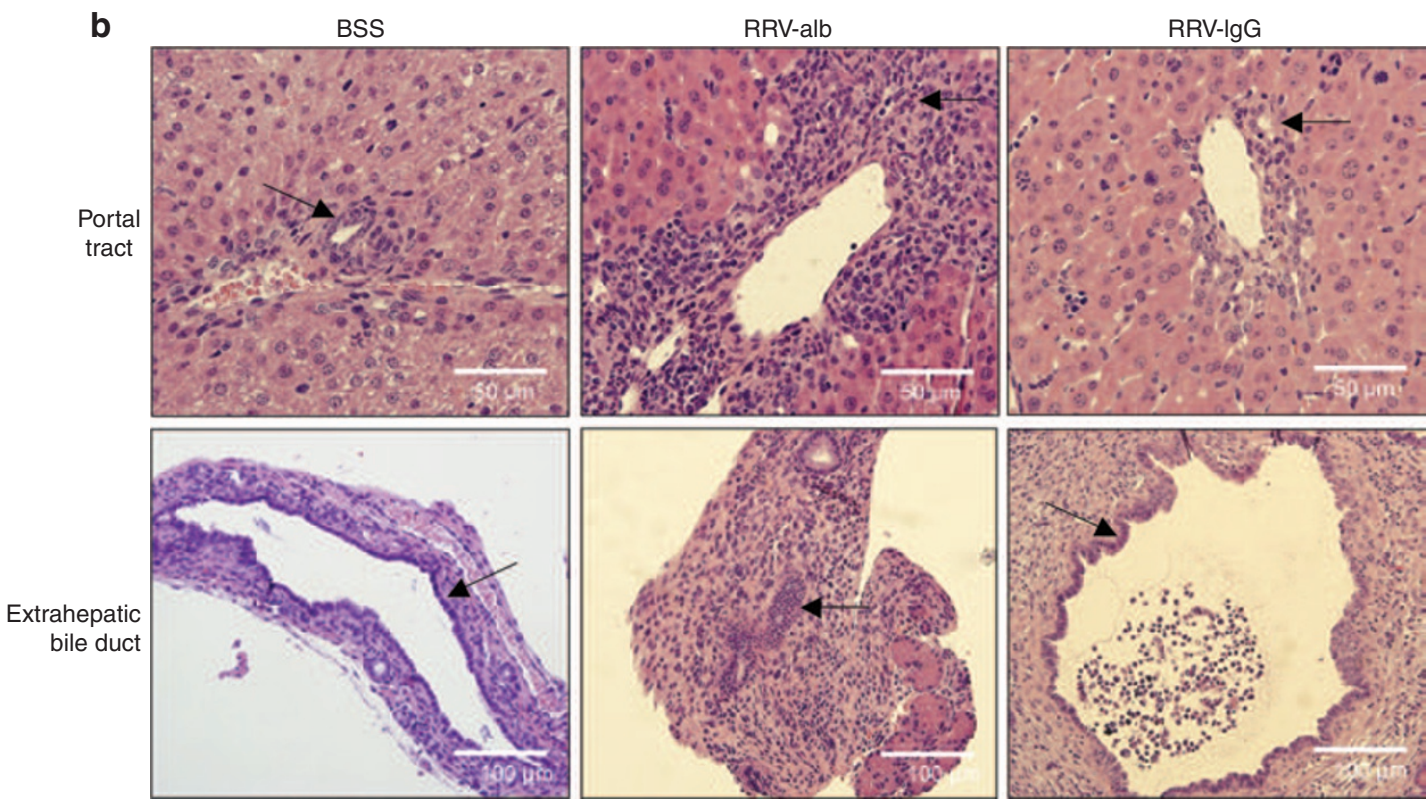

Figure 2. Biliary obstruction. (a) Serum direct bilirubin. Shown is the mean \pm SEM of serum direct bilirubin levels obtained at $14 \mathrm{~d}$ of life, demonstrating significant bilirubin reduction in RRV-lgG mice $\left.{ }^{*} P<0.001 * * *<0.0001\right)$. (b) Liver and extrahepatic bile duct histology. Shown is hematoxylin and eosin histology from intrahepatic (portal tract $\times 400)$ and extrahepatic bile ducts $(\times 200)$ (arrows indicate bile ducts). Note diminished portal periductal inflammation and lack of extrahepatic biliary obstruction in the RRV-IgG group. BSS, balanced salt solution; RRV, rhesus rotavirus.

mice. (Figure 3c) The lack of inflammation in the portal tracts correlated with the markedly diminished periductal inflammation identified by hematoxylin and eosin histology (Figure 2b).

In order to investigate the mechanism associated with the lack of portal tract inflammation in the RRV-IgG mice, chemokine and adhesion molecules essential for trafficking of immune cells to sites of injury were analyzed in the liver. There was no significant decrease in chemokine levels of expression in RRV-IgG mice compared to other RRV groups (Supplementary Figure S2a online). Adhesion molecule analysis revealed significantly increased levels of vascular cell adhesion molecule 1 (VCAM-1) after RRV infection. VCAM-1 expression was significantly decreased in RRV-IgG mice compared to BA-RRV $(P=0.02)$ and RRV-albumin groups $(P=$ 0.001) (Figure 4a). Importantly, immunohistochemistry studies of VCAM-1 protein expression within the liver demonstrated substantially diminished VCAM-1 within the portal tracts of RRV-infected mice that received high-dose IgG therapy (Figure $4 \mathrm{~b}$ ). VCAM-1 is known to be expressed on endothelium and promotes the adhesion of lymphocytes and monocytes, mediating both cell migration and activation (25). High-dose IgG therapy diminished VCAM-1 expression within portal tracts of RRV-infected mice, thereby inhibiting infiltration of activated immune cells.

\section{High-Dose IgG Therapy Resulted in Significantly Decreased Production of Inflammatory Cytokines From CD4 ${ }^{+} \mathrm{T}$ Cells}

High-dose IgG is known to inhibit proinflammatory Th1 cytokine production $(26,27)$. Therefore, we next addressed the question of potential changes in effector cell cytokine production in RRV-IgG mice. Intracellular cytokine production from liver immune cells of 10-d-old mice included analysis of interleukin (IL)-2, IL-17, interferon (IFN)- $\gamma$, and tumor necrosis factor (TNF)- $\alpha$ after phorbol 12-myristate 13-acetate (PMA)/ionomycin stimulation (Figures 5 and 6). Compared to BSS controls, BA-RRV mice had marked 
a

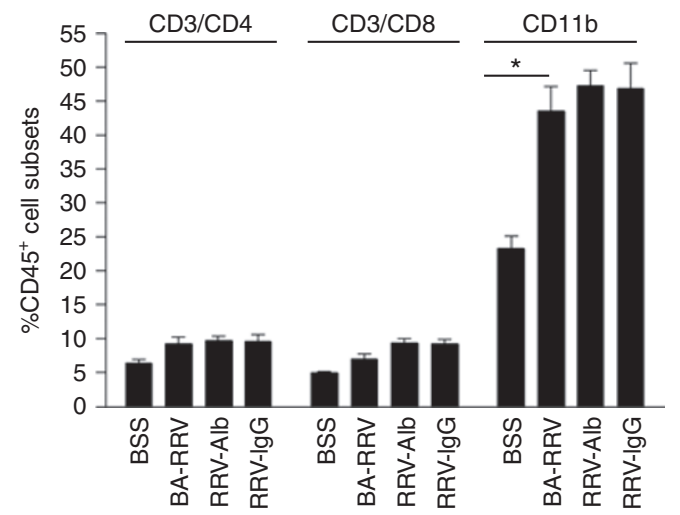

C

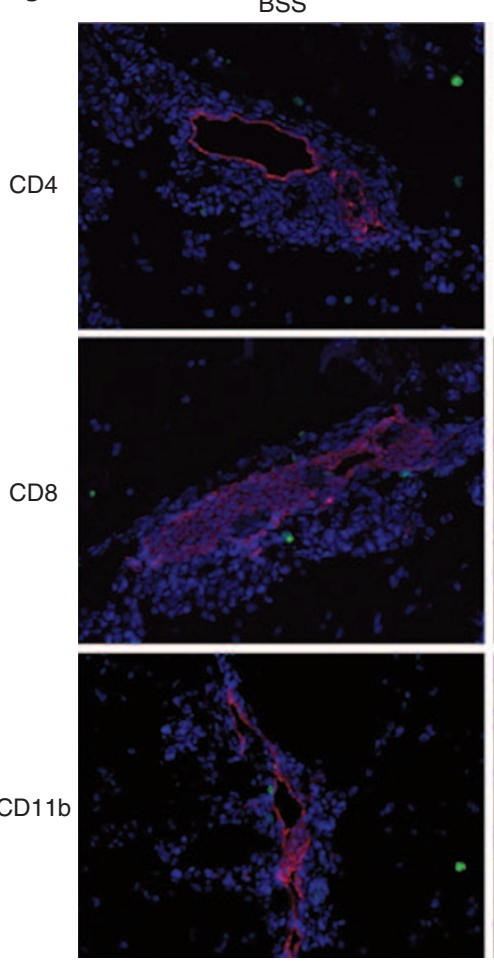

BA-RRV

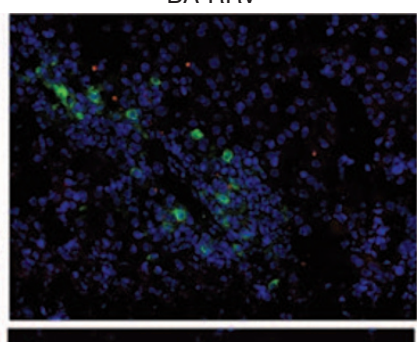

b

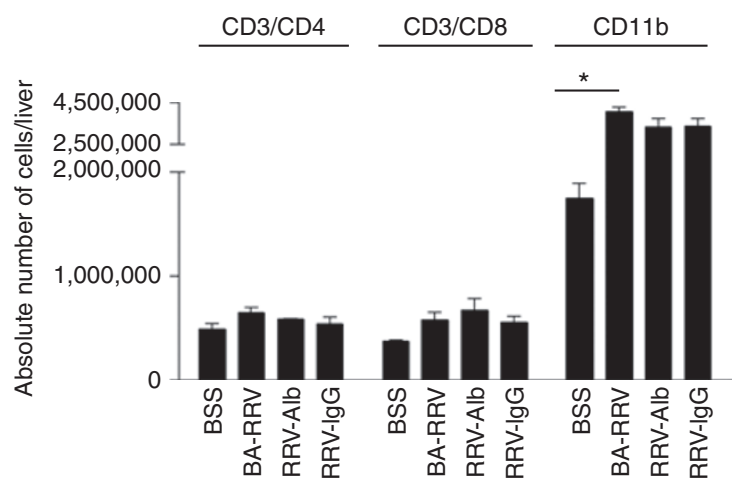

RRV-Alb
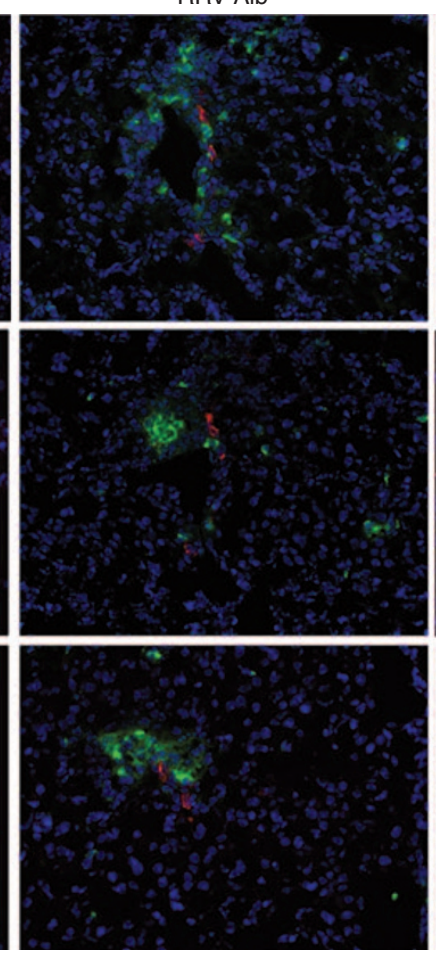

RRV-IgG
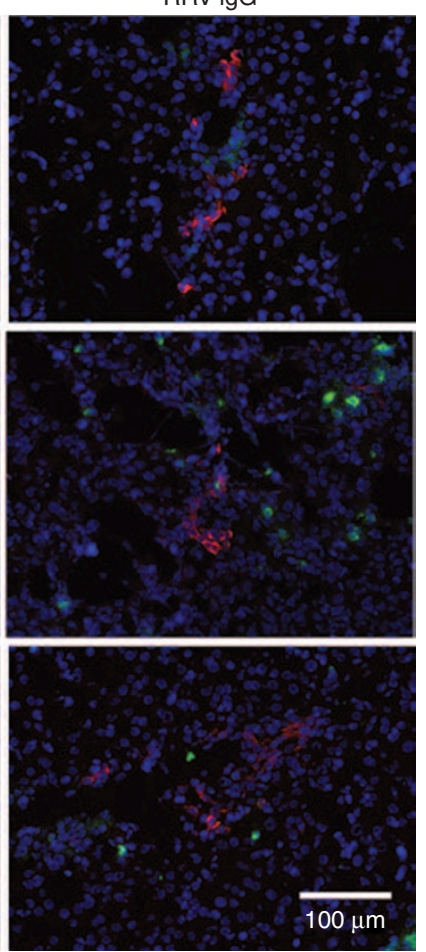

Figure 3. RRV-lgG mice have similar immune cell profile yet diminished bile duct-targeted inflammation. Fluorescence-activated cell sorting (FACS) analysis of total liver immune cells from 10-d-old mice. Quantification of each immune cell type is expressed as the (a) percent or the (b) absolute numbers of $\mathrm{CD}_{4} 5^{+}$cells that were also positive for the specific immune cell subset markers (shown as mean $\pm \mathrm{SEM}$ ). The total liver immune cell profile in all RRV-infected groups was similar and all cell lines were increased compared to the BSS control group $\left({ }^{*} P<0.005\right)$. (c) Fluorescent immunohistochemistry $(\times 200)$ of portal tract immune cells. Liver tissue was stained with AlexaFlor555 anti-TROMA (red) to identify bile duct epithelia, and double stained with either fluorescein isothiocyanate (FITC) anti-CD4, -CD8, or -CD11b (green) and nuclear counterstain with Hoescht (blue). Inflammatory cell infiltrates are present in the portal tracts of BA-RRV and RRV-alb mice but were attenuated in RRV-lgG mice. BA, biliary atresia; BSS, balanced salt solution; RRV, rhesus rotavirus.

increases in $\mathrm{CD}^{+} \mathrm{T}$ cells producing IL-2 (BSS $0.25 \pm 0.003 \%$; BA-RRV 2.95 $\pm 0.52 \%, P=0.002)$, TNF- $\alpha$ (BSS $0.20 \pm 0.05 \%$; BA-RRV 29.30 $\pm 5.18 \%, P<0.0001$ ), and IFN- $\gamma$ (BSS $0.17 \pm 0.05 \%$; BA-RRV: $20.93 \pm 3.38 \%, P<0.0001)$ and in $\mathrm{CD}^{+} \mathrm{T}$ cells producing IFN- $\gamma$ (BSS $0.25 \pm 0.002 \%$; BA-RRV 30.16 $\pm 4.99 \%, P=0.0003)$. TNF- $\alpha$ production by

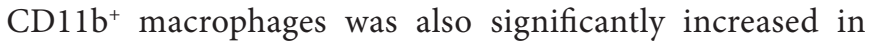
BA-RRV mice compared to BSS (BSS 0.39 $\pm 0.06 \%$; BA-RRV $5.59 \pm 1.05 \%, P=0.002)$. Immune cells from RRV-alb mice generated similar proinflammatory cytokine levels as the BA-RRV mice. In stark contrast, RRV-IgG mice had significantly reduced $\mathrm{CD}^{+} \mathrm{T}$ cell production of IL-2 (RRV-alb
$4.21 \pm 1.00 \% ;$ RRV-IgG $1.27 \pm 0.23 \%, P=0.0036)$, IFN- $\gamma$ (RRV-alb $31.65 \pm 1.69 \%$; RRV-IgG $15.33 \pm 1.75 \%, P=0.0015)$ and TNF- $\alpha$ (RRV-alb 27.10 $\pm 3.13 \%$; RRV-IgG 9.26 $\pm 0.62 \%$, $P=0.007)$ and in $\mathrm{CD}^{+} \mathrm{T}$ cells producing IFN- $\gamma(\mathrm{RRV}$-alb $31.15 \pm 6.51 \%$; RRV-IgG $14.73 \pm 2.44 \%, P=0.029)$. There was no effect of high-dose IgG on $\mathrm{CD}^{+} \mathrm{T}$ cell $\mathrm{IL}-17$ production (RRV-alb $0.2 \pm 0.13 \%$; RRV-IgG $0.3 \pm 0.16 \%, P>0.05$ ).

\section{High-Dose IgG Therapy Was Associated With Liver Treg Expansion}

We next sought to determine whether the mechanism by which IgG reduced bile duct inflammation and cytokine 

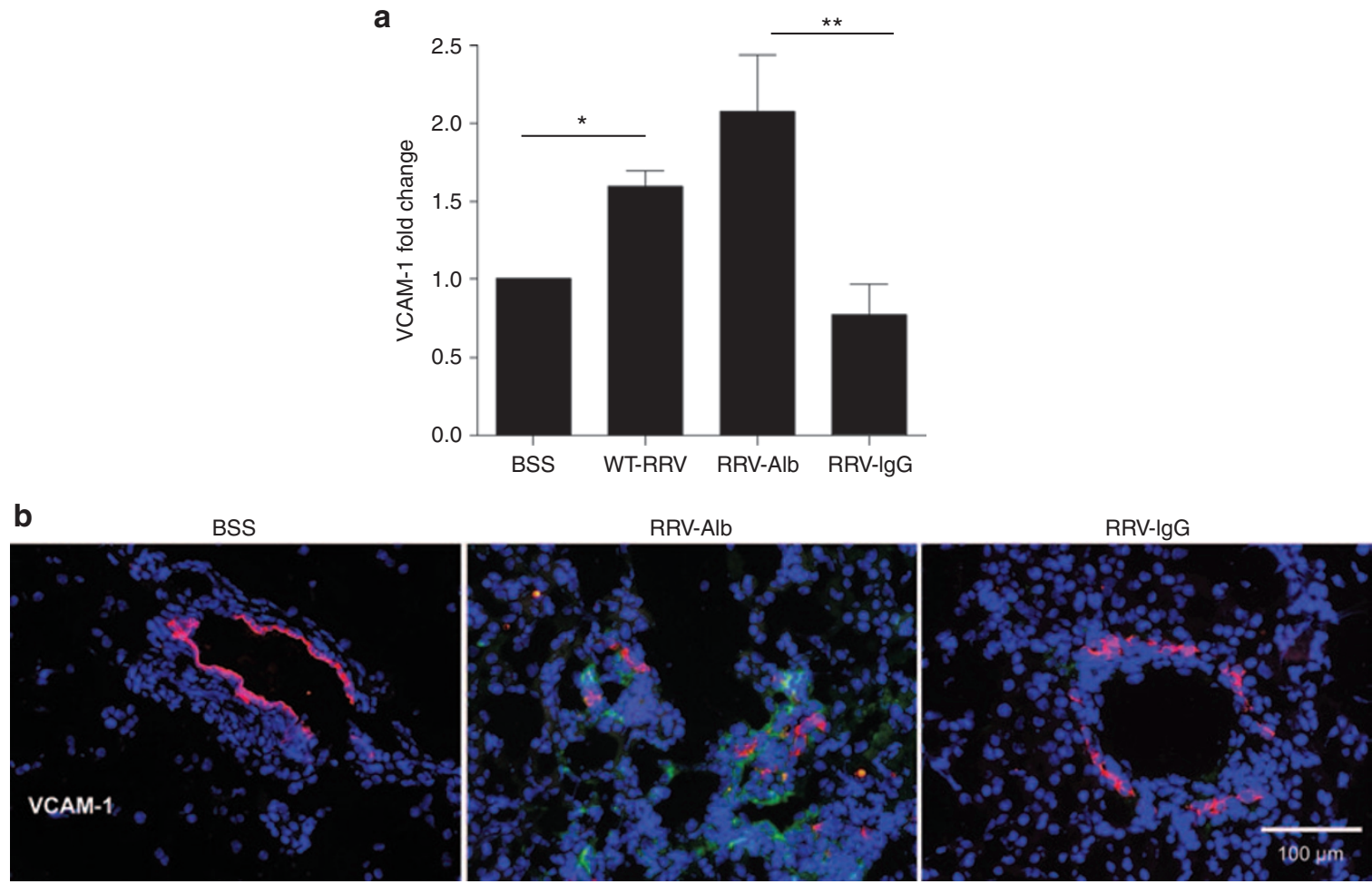

Figure 4. Vascular cell adhesion molecule 1 (VCAM-1) is downregulated following high dose lgG treatment. (a) VCAM-1 mRNA expression. Total RNA was isolated from whole livers and VCAM-1 mRNA expression was ascertained in relation to the housekeeping gene actin. RRV-alb mice had significantly increased VCAM-1 expression compared to the BSS controls and high-dose lgG treatment led to a significant reduction in VCAM- 1 expression $\left({ }^{*} P<0.05\right.$, ${ }^{*} P<<0.005$ ). (b) VCAM protein expression. Liver tissue was stained with AlexaFlor555 anti-TROMA (bile ducts (red)), fluorescein isothiocyanate (FITC) anti-VCAM (green), and nuclear Hoescht (blue). Shown is representative portal tract ( $\times 200)$ VCAM-1 expression. VCAM-1 expression is present in the portal tracts of RRV-alb mice but was not observed in BSS or RRV-IgG mice. BSS, balanced salt solution; RRV, rhesus rotavirus.

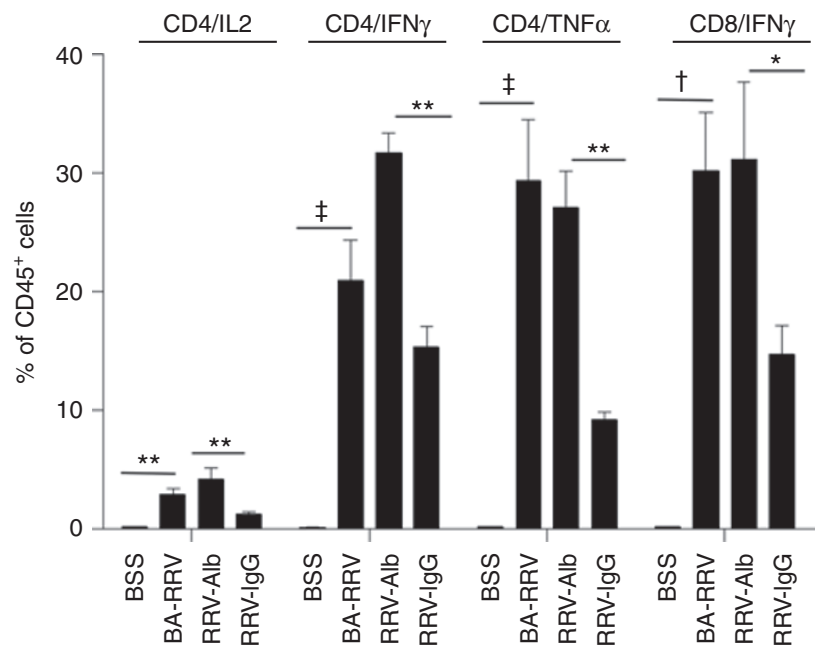

Figure 5. Marked attenuation of $\mathrm{CD} 4^{+} \mathrm{Th} 1$ cytokine production with high-dose IgG therapy. Summary of intracellular cytokine production. Liver immune cells were stained with cell surface markers (CD45.2 and $\mathrm{CD} 3 / \mathrm{CD} 4, \mathrm{CD} 3 / \mathrm{CD} 8$ or $\mathrm{CD} 11 \mathrm{~b})$, stimulated with phorbol 12-myristate 13 -acetate (PMA)/ionomycin and interleukin (IL)-2, interferon (IFN)- $\gamma$, tumor necrosis factor (TNF)- $\alpha$, and IL-17 quantified by intracellular staining and fluorescence-activated cell sorting (FACS) analysis. High-dose IgG treatment was associated with significantly reduced inflammatory cytokine production by CD4 ${ }^{+}$T cells $\left({ }^{*} P<0.05,{ }^{* *} P<0.005,{ }^{+} P<0.0005\right.$, $\left.{ }^{\ddagger} P<0.0001\right)$. BA, biliary atresia; BSS, balanced salt solution; RRV, rhesus rotavirus. production involved changes in Tregs. Tregs are defined based on coexpression of $\mathrm{CD} 25^{+} \mathrm{FoxP}^{+}{ }^{+}$within the $\mathrm{CD} 4^{+}$ $\mathrm{T}$ cell subset and are potent inhibitors of $\mathrm{T}$ cell-mediated inflammation. A significant reduction in the percent of $\mathrm{CD}_{25}{ }^{+} \mathrm{FoxP}_{3}{ }^{+}$Tregs within the $\mathrm{CD}^{+} \mathrm{T}$ cell subset was observed in 10-d-old BA-RRV mice compared to BSS control mice (BSS 9.38 $\pm 0.49 \%$; BA-RRV $5.75 \pm 0.52 \%, P=0.0003$ ). Importantly, the RRV-IgG mice had significantly increased $\mathrm{CD} 4^{+} \mathrm{CD} 25^{+} \mathrm{FoxP}^{+}$Tregs compared to the RRV-albumin group (RRV-alb $7.51 \pm 0.03 \%$; RRV-IgG $10.25 \pm 0.75 \%, P$ $=0.0035)$ and were comparable to the BSS control mice. (Figure 7a,b) Thus, IgG therapy was associated with a significant expansion of Tregs in RRV-infected BA mice, restoring Treg numbers back to that of BSS controls.

\section{DISCUSSION}

Novel therapeutic approaches targeted to suppress the inflammatory response are warranted to delay or avoid the need for liver transplantation in BA. To this end, this study demonstrated the proof of principle that high-dose IgG could reduce intrahepatic and extrahepatic bile duct inflammation and injury in the RRV-induced mouse model of BA. This effect is associated with concomitant expansion of liver Tregs and attenuation of VCAM-1 expression, portal tract inflammation, and $\mathrm{CD} 4^{+}$Th1 cytokine production (Figure 8). 

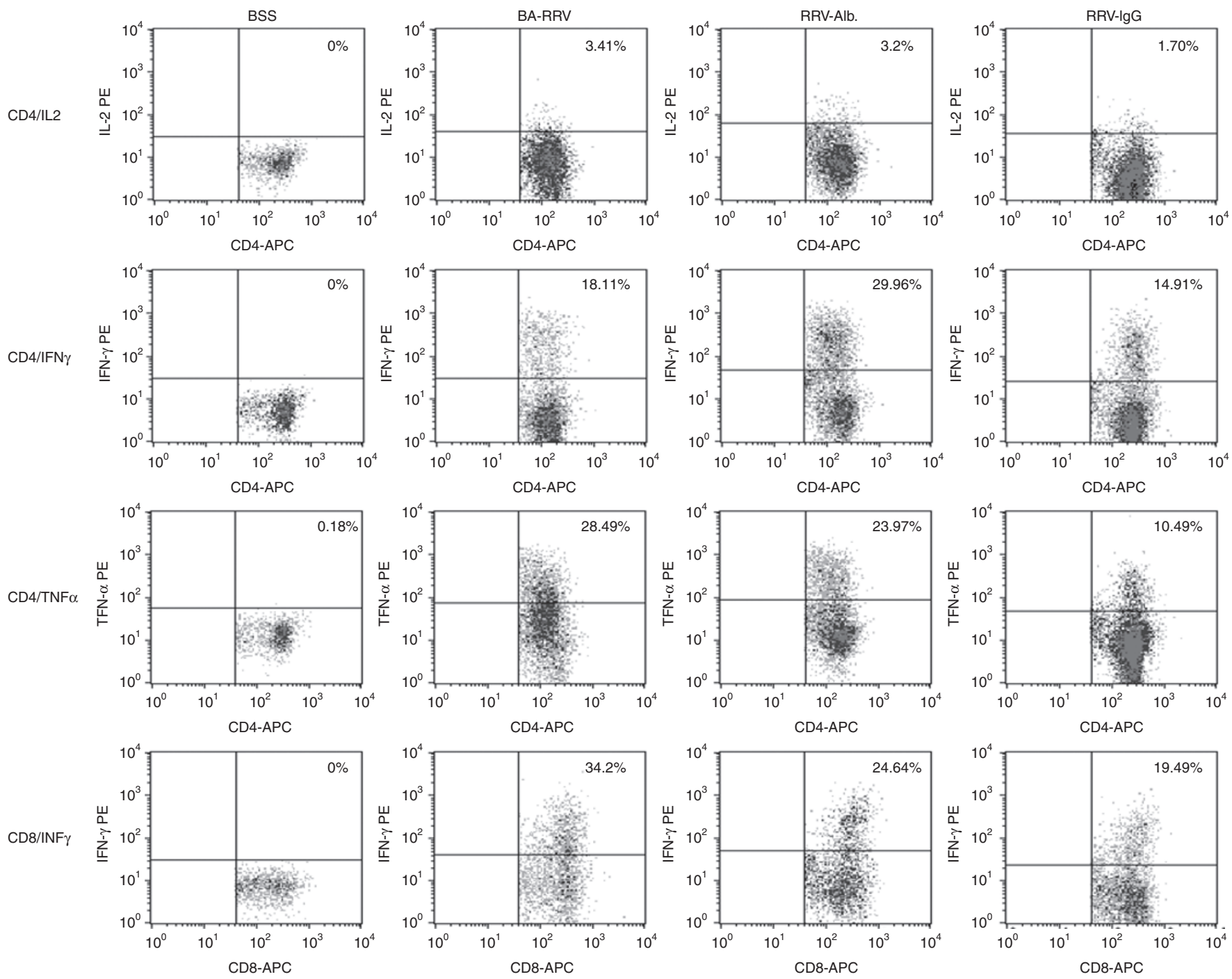

Figure 6. Representative fluorescence-activated cell sorting (FACS) analysis dot plot of cytokine production. Shown is an example of intracellular cytokine staining from CD4 ${ }^{+}$and $\mathrm{CD}^{+} \mathrm{T}$ cells following phorbol 12-myristate 13-acetate (PMA)/ionmycin stimulation. Quadrant marker placement was established based on the negative cell population. Leukocyte populations were identified for study by triple gating (details on gating in Supplementary Figure S3 online). BA, biliary atresia; BSS, balanced salt solution; RRV, rhesus rotavirus.

One limitation to this study is that despite inhibition of inflammation and biliary obstruction, survival was not significantly improved with high-dose IgG. The lack of increased survival in the RRV-IgG mice was not due to persistent virus infection, as the RRV-IgG mice cleared virus as effectively as other RRV-infected controls. It is likely that RRV-infected mice were already too ill at the time of initial IgG treatment to have any mortality benefit from high-dose IgG therapy. This study as well as previous reports have shown that RRV-infected BA mice weigh less than BSS controls, reflecting decreased oral intake, malabsorption, and probable neglect by the mother $(9,10)$. This deficit in growth was not reversed by IgG therapy and most likely contributed to mortality. Another possibility is that the RRV-IgG mice had the equivalent of serum sickness, from the combination of circulating human IgG and anti-RRV antibodies. However, the control group of BSS mice that received IgG had 100\% survival, confirming that administration of human IgG (without infection) was not harmful. Human IgG was employed instead of mouse IgG, because human IgG has been previously shown to have high affinity for the neonatal Fc receptor in mice $(28,29)$. Furthermore, the majority of previous studies examining the effect of IgG in mouse models of human disease have employed human IgG preparations (20-23). Despite the finding that high-dose IgG did not change survival, the marked inhibition of bile duct inflammation and obstruction suggests that IgG therapy could have a potential benefit in BA infants who undergo the Kasai procedure.

One mechanism by which high-dose IgG may reduce portal tract inflammation is through inhibition of VCAM-1. Through its interaction with the very-late activation 4 ligand expressed on monocytes and T and B lymphocytes, VCAM-1 promotes adhesion and transendothelial migration of inflammatory cells into injured tissue (30). Increased VCAM-1 expression in several inflammatory liver processes has been reported, particularly 


\section{Articles | Fenner etal.}

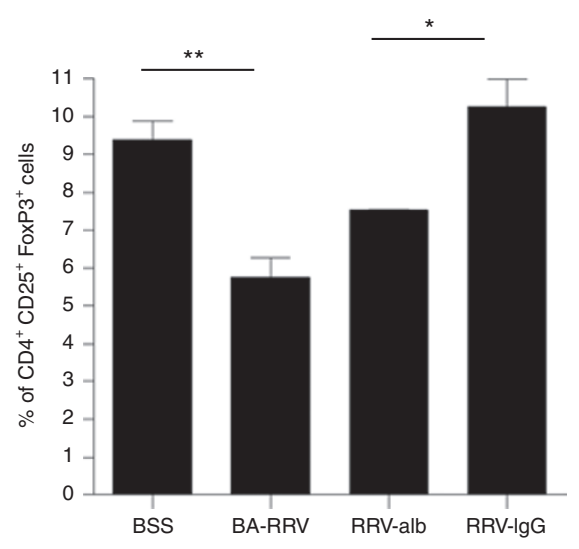

b

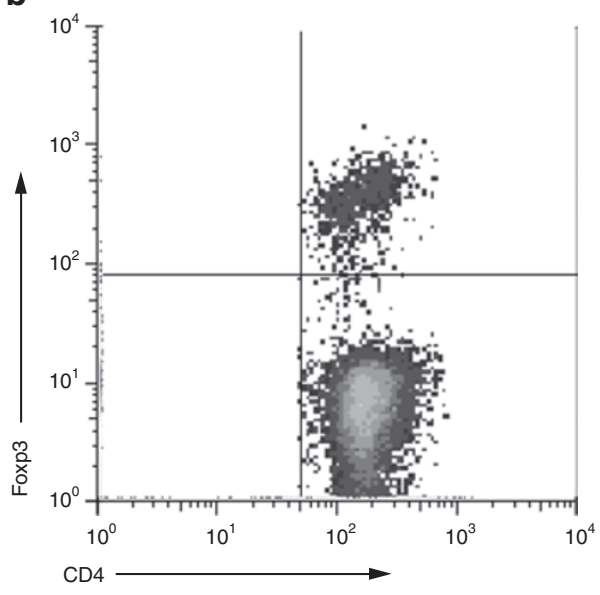

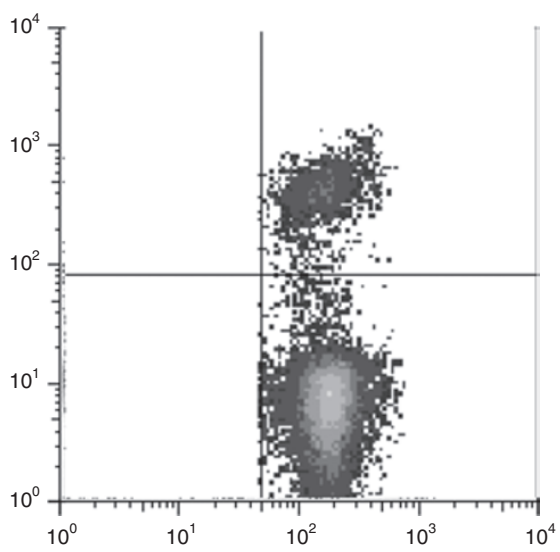

Figure 7. High-dose IgG is associated with liver Treg expansion. (a) Summary of fluorescence-activated cell sorting (FACS) analysis of liver Tregs. Percoll gradient-purified liver immune cells were stained with CD4, CD25, and FoxP3 to identify Tregs $\left({ }^{*} P<0.005\right.$, $\left.{ }^{* *} P<0.0005\right)$. (b) Representative dot plots. Shown are representative dot plots of $\mathrm{CD}^{+} \mathrm{FoxP3}^{+}$Tregs gated on liver $\mathrm{CD} 4^{+}$cells. Left panel—RRV-alb: $7.4 \%$ Tregs, right panel—RRV-lgG: 10.5\% Tregs. BA, biliary atresia; BSS, balanced salt solution; RRV, rhesus rotavirus; Tregs, regulatory T cells.
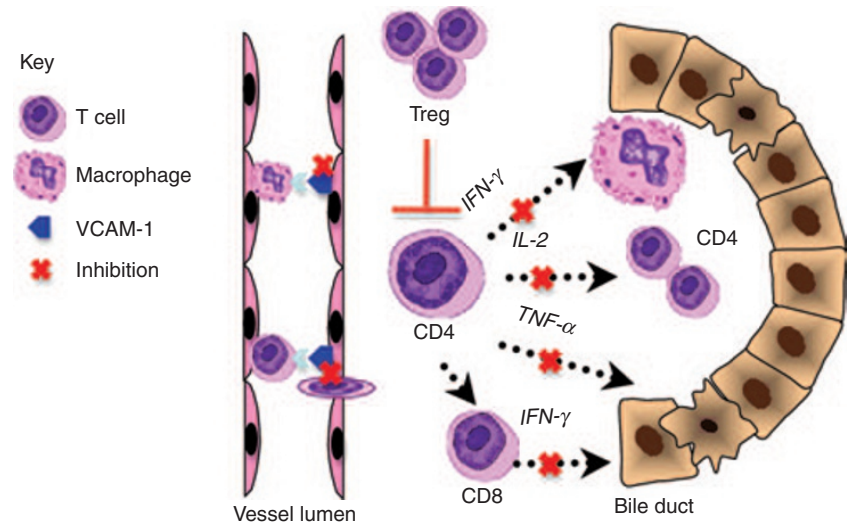

Figure 8. Mechanisms of action of high-dose lgG in murine biliary atresia (BA). Shown is the summary of effects of high-dose lgG in murine BA, including inhibition of vascular cell adhesion molecule 1 expression on vascular endothelium, resulting in decreased portal tract extravasation of T cells and monocytes; inhibition of Th1 cell activation, leading to decreased interferon (IFN)- $\gamma$-associated macrophage stimulation, interleukin (IL)-2-associated T cell expansion, cytotoxic tumor necrosis factor (TNF)- $\alpha$ production and $\mathrm{CD}^{+}{ }^{+} \mathrm{T}$ cell-mediated stimulation of cytotoxic $\mathrm{CD}^{+} \mathrm{T}$ cells; and expansion of Tregs resulting in T cell inhibition. Treg, regulatory T cell; VCAM-1, vascular cell adhesion molecule 1.

in those characterized by periportal inflammation $(31,32)$. Kobayashi et al. (33). previously identified increased expression of VCAM-1 on bile ducts, endothelial cells, and hepatocytes from human patients with severe BA compared to healthy controls and patients with less severe BA. In the mouse model of BA, high-dose IgG inhibited VCAM-1 expression, thereby reducing trafficking of lymphocytes and monocytes into the portal tracts. VCAM-1 expression by endothelial cells is stimulated by inflammatory cytokines, namely TNF- $\alpha$, and inhibition of VCAM- 1 by high-dose IgG may be related to its concurrent inhibitory effects on inflammatory cytokine production $(30,34)$.

This study demonstrated an overproduction of the proinflammatory cytokines IL- 2 , IFN- $\gamma$, and TNF- $\alpha$ by hepatic CD $4^{+}$
T cells and IFN- $\gamma$ by $C D 8^{+} \mathrm{T}$ cells from RRV-infected mice that was dampened by high-dose IgG therapy. IFN- $\gamma$-producing $T$ cells have been implicated in the pathology of several inflammatory autoimmune diseases (35). In the mouse model of BA, Shivakumar et al. (10) demonstrated the essential role of IFN- $\gamma$ in bile duct injury, as IFN- $\gamma$ knockout mice infected with RRV had resolution of biliary obstruction and improved survival. In the current study, high-dose IgG therapy was associated with significant reduction in IFN- $\gamma$ and protection from biliary injury. This is in accordance with previous studies on inflammatory and autoimmune diseases that have shown that highdose IgG therapy reduces levels of IFN- $\gamma$, TNF- $\alpha$, and other inflammatory cytokines $(22,36)$. IFN- $\gamma$ has many downstream effects, including activation of macrophages with subsequent release of cytotoxic molecules. This study showed that the diminished levels of IFN- $\gamma$ in the RRV-IgG group correlated with decreased portal tract macrophage infiltrates and TNF- $\alpha$ producing macrophages, suggesting a synergistic mechanism of protection from bile duct injury. It has been hypothesized that the reduction of cytokine production by IgG therapy may be due to antibodies contained in polyclonal IgG that inactivate these cytokines $(37,38)$, by direct antibody neutralization of antigens (39) or through the enhancement of the inhibitory action of Tregs (40).

Tregs are important in suppressing the $\mathrm{T}$ cell-mediated immune response to pathogens, inhibiting overly aggressive immune activation and preventing activation of autoreactive $\mathrm{T}$ cells. Other studies have shown that the beneficial effects of high-dose IgG were linked to expansion of Treg populations. In murine experimental autoimmune encephalomyelitis, a model of multiple sclerosis, Ephrem et al. (40) observed that prophylactic dosing of high-dose IgG therapy prevented experimental autoimmune encephalomyelitis in mice and was associated with increased Treg numbers and suppressive capacity. The expansion of liver Tregs identified in our study offers a unifying mechanism as to how high-dose IgG 
reduced proinflammatory cytokine production by $\mathrm{CD} 4^{+}$and $\mathrm{CD}^{+}$effector $\mathrm{T}$ cells and diminished biliary tract injury. The potential importance of Tregs in the pathogenesis of BA has recently been described. Our group reported that circulating Tregs were significantly lower in infants with BA at the time of diagnosis compared to age-matched controls (15). Likewise, in BA mouse studies, Treg deficits have been reported as well as protection from BA with Treg supplementation $(14,16)$. Given these findings, developing therapies that stimulate Tregs might have significant impact on the outcomes of human BA.

In conclusion, we have demonstrated that administration of high-dose IgG attenuated bile duct inflammation and injury in the mouse model of BA. Our observations should encourage the development of clinical trials of high-dose IVIg as an adjuvant therapy to Kasai portoenterostomy in infants with BA. In combination with surgery, IVIg might have the potential to change the trajectory of the disease and delay or obviate the need for liver transplantation.

\section{METHODS}

\section{RRV-Induced Mouse Model of BA and IgG Treatment}

Timed-pregnant female BALB/c mice were purchased from rotavirus-free colonies of Jackson Laboratory (Bar Harbor, ME). Mice were injected intraperitoneally at $12-18 \mathrm{~h}$ of life with either $1.5 \times 10^{6} \mathrm{pfu} / \mathrm{ml}$ of RRV or BSS as control. RRV-infected, jaundiced mice received an intraperitoneal injection of either $2 \mathrm{~g} / \mathrm{kg}$ polyclonal, human IgG (Gammagard, Baxter, Westlake Village, CA), human albumin (Sigma Aldrich, St Louis, MO), or no treatment every 2-3 d × 6 doses, starting on day 7 of life. On days 10-14, serum, liver, and extrahepatic bile ducts were harvested. All animals were handled in accordance with the National Institutes of Health "Guide for Care and Use of Laboratory Animals" (publication \#86-25 1985) through the University of Colorado Denver Office of Laboratory Animal Medicine.

\section{Viral Plaque Assay}

Liver tissue was homogenized in sterile MEM (1:1 weight/volume) and plated on a monolayer of MA-104 cells as previously described (9).

\section{Serum Bilirubin Assay}

Blood was collected from the renal artery, pooled (2-4 mice/pool), and serum direct bilirubin was measured with direct bilirubin assay (Diagnostic Chemicals, Oxford, CT).

\section{Histopathology}

Formalin-fixed, paraffin-embedded liver and extrahepatic bile duct sections ( $n=6$ each group) were stained with hematoxylin and eosin and visualized with the Olympus BX41 Laboratory Microscope (Olympus America, Scientific Equipment Group, Center Valley, PA).

\section{Indirect Immunofluorescence}

Snap frozen liver tissue ( $n=6$ each group) was stained using Tyramide System Amplification Direct Kit (PerkinElmer, Waltham, MA) according to manufacturer's instructions. Slides were incubated with anti-TROMA III (CK19 on bile duct epithelium) and either antibodies to CD4 (GK1.5), CD8a (5H10), CD11b (M1/70), VCAM-1 (429), anti-rat IgG2a (eBM2a), or anti-rat IgG2b (eB149/10H5) (eBiosciences, San Diego, CA) and counterstained with Hoechst nuclear stain (Sigma, St Louis, MO). Fluorescent signal was photographed with the Zeiss Axio Imager.A1 (Carl Zeiss, Thornwood, NY) and layers were overlaid using Adobe Photoshop CS2 (Adobe Systems, San Jose, CA).

\section{Flow Cytometry of Hepatic Immune Cells}

Two to four livers were pooled for isolation of liver immune cells and $\geq 3$ pools were analyzed for each experiment. Single cell suspension of livers were stained with anti-CD45, anti-CD3 and anti-CD4, CD8, CD11b, NKG2D, B220, or isotype controls (eBiosciences). For liver Treg analysis, immune cells were isolated by Percoll gradient and stained using the Mouse Regulatory T cell Staining Kit per manufacturer's instructions (eBiosciences). Expression of cell surface markers was determined with the FACSCalibur flow cytometer using CELLQuest software (Becton-Dickinson, San Jose, CA) for analysis. Leukocyte populations were identified by gating on $\mathrm{CD} 45^{+}$cells.

\section{Intracellular Cytokine Analysis by Flow Cytometry}

Hepatic immune cells were stimulated with phorbol 12-myristate 13-acetate (PMA) and ionomycin in GolgiPlug (BD Pharmingen, San Diego, CA), incubated with anti-CD45.2 (cells of hematopoietic origin) and either anti-CD4, CD8a, or CD11b (eBiosciences). Cells were permeabilized and incubated with either anti-IL-2, IFN- $\gamma$, TNF- $\alpha$, IL-17, or isotype control (eBiosciences).

\section{Isolation of Liver RNA and qRT-PCR}

Liver tissue was homogenized in TRIZOL buffer (Invitrogen, Carlsbad, CA) and RNA isolated according to manufacturer's protocol. RT-PCR was performed using TaqMan primers (Invitrogen) and qScript 1-Step Fast qRT-PCR Kit (Quanta Biosciences, Gaithersburg, MD) on an iCycler thermal cycler (BioRad, Hercules, CA).

\section{Statistical Analysis}

All results were based on $\geq 3$ separate experiments of pooled liver samples (2-4 samples/pool) and shown as mean \pm SEM. Differences between groups were analyzed with the one-way ANOVA and adjusted using the Šidák correction (Prism, GraphPad Software, La Jolla, CA). Differences in means were considered significant for $P$ values $<0.05$.

\section{SUPPLEMENTARY MATERIAL}

Supplementary material is linked to the online version of the paper at http://www.nature.com/pr

\section{STATEMENT OF FINANCIAL SUPPORT}

This study was supported by National Institutes of Health; National Institute of Diabetes and Digestive and Kidney Diseases, R01:DK078195 (PI: Mack CL); CHCO Pediatric Liver Research Fund, Aurora, CO; and CHCO Research Institute, Aurora, CO.

Disclosure: There are no financial interests of any of the authors that could be perceived as being a conflict of interest.

\section{REFERENCES}

1. Davenport M. Biliary atresia: clinical aspects. Semin Pediatr Surg 2012;21:175-84.

2. Jimenez-Rivera C, Jolin-Dahel KS, Fortinsky KJ, Gozdyra P, Benchimol EI. International incidence and outcomes of biliary atresia. J Pediatr Gastroenterol Nutr 2013;56:344-54.

3. Gallo A, Esquivel CO. Current options for management of biliary atresia. Pediatr Transplant 2013;17:95-8.

4. Petersen C, Davenport M. Aetiology of biliary atresia: what is actually known? Orphanet J Rare Dis 2013;8:128.

5. Bessho K, Bezerra JA. Biliary atresia: will blocking inflammation tame the disease? Annu Rev Med 2011;62:171-85.

6. Mack CL, Feldman AG, Sokol RJ. Clues to the etiology of bile duct injury in biliary atresia. Semin Liver Dis 2012;32:307-16.

7. Riepenhoff-Talty M, Schaekel K, Clark HF, et al. Group A rotaviruses produce extrahepatic biliary obstruction in orally inoculated newborn mice. Pediatr Res 1993;33(4 Pt 1):394-9.

8. Mack CL, Tucker RM, Sokol RJ, et al. Biliary atresia is associated with CD4+ Th1 cell-mediated portal tract inflammation. Pediatr Res 2004;56:79-87.

9. Mack CL, Tucker RM, Sokol RJ, Kotzin BL. Armed CD4+ Th1 effector cells and activated macrophages participate in bile duct injury in murine biliary atresia. Clin Immunol 2005;115:200-9.

10. Shivakumar P, Campbell KM, Sabla GE, et al. Obstruction of extrahepatic bile ducts by lymphocytes is regulated by IFN- $\gamma$ in experimental biliary atresia. J Clin Invest 2004;114:322-9.

11. Mack CL, Tucker RM, Lu BR, et al. Cellular and humoral autoimmunity directed at bile duct epithelia in murine biliary atresia. Hepatology 2006;44:1231-9. 
12. Terrier B, Degand N, Guilpain P, Servettaz A, Guillevin L, Mouthon L. Alpha-enolase: a target of antibodies in infectious and autoimmune diseases. Autoimmun Rev 2007;6:176-82.

13. Lu BR, Brindley SM, Tucker RM, Lambert CL, Mack CL. a-enolase autoantibodies cross-reactive to viral proteins in a mouse model of biliary atresia. Gastroenterology 2010;139:1753-61.

14. Lages CS, Simmons J, Chougnet CA, Miethke AG. Regulatory T cells control the CD8 adaptive immune response at the time of ductal obstruction in experimental biliary atresia. Hepatology 2012;56:219-27.

15. Brindley SM, Lanham AM, Karrer FM, Tucker RM, Fontenot AP, Mack CL. Cytomegalovirus-specific T-cell reactivity in biliary atresia at the time of diagnosis is associated with deficits in regulatory $\mathrm{T}$ cells. Hepatology 2012;55:1130-8.

16. Tucker RM, Feldman AG, Fenner EK, Mack CL. Regulatory T cells inhibit Th1 cell-mediated bile duct injury in murine biliary atresia. J Hepatol 2013;59:790-6.

17. Kazatchkine MD, Kaveri SV. Immunomodulation of autoimmune and inflammatory diseases with intravenous immune globulin. N Engl J Med 2001;345:747-55.

18. Rauova L, Rovensky J, Shoenfeld Y. Immunomodulation of autoimmune diseases by high-dose intravenous immunoglobulins. Springer Semin Immunopathol 2001;23:447-57.

19. Gelfand EW. Intravenous immune globulin in autoimmune and inflammatory diseases. N Engl J Med 2012;367:2015-25.

20. Samuelsson A, Towers TL, Ravetch JV. Anti-inflammatory activity of IVIG mediated through the inhibitory Fc receptor. Science 2001;291:484-6.

21. Zhu KY, Feferman T, Maiti PK, Souroujon MC, Fuchs S. Intravenous immunoglobulin suppresses experimental myasthenia gravis: immunological mechanisms. J Neuroimmunol 2006;176:187-97.

22. Kishimoto C, Takada H, Kawamata H, Umatake M, Ochiai H. Immunoglobulin treatment prevents congestive heart failure in murine encephalomyocarditis viral myocarditis associated with reduction of inflammatory cytokines. J Pharmacol Exp Ther 2001;299:645-51.

23. Achiron A, Mor F, Margalit R, Cohen IR, Lider O, Miron S. Suppression of experimental autoimmune encephalomyelitis by intravenously administered polyclonal immunoglobulins. J Autoimmun 2000;15:323-30.

24. Maddur MS, Othy S, Hegde P, et al. Immunomodulation by intravenous immunoglobulin: role of regulatory T cells. J Clin Immunol 2010;30:Suppl $1: S 4-8$.

25. Gearing AJ, Newman W. Circulating adhesion molecules in disease. Immunol Today 1993;14:506-12.

26. Achiron A, Margalit R, Hershkoviz R, et al. Intravenous immunoglobulin treatment of experimental $\mathrm{T}$ cell-mediated autoimmune disease. Upregulation of $\mathrm{T}$ cell proliferation and downregulation of tumor necrosis factor alpha secretion. J Clin Invest 1994;93:600-5.
27. Andersson J, Skansén-Saphir U, Sparrelid E, Andersson U. Intravenous immune globulin affects cytokine production in $\mathrm{T}$ lymphocytes and monocytes/macrophages. Clin Exp Immunol 1996;104:Suppl 1:10-20.

28. Ober RJ, Radu CG, Ghetie V, Ward ES. Differences in promiscuity for antibody-FcRn interactions across species: implications for therapeutic antibodies. Int Immunol 2001;13:1551-9.

29. Andersen JT, Daba MB, Berntzen G, Michaelsen TE, Sandlie I. Crossspecies binding analyses of mouse and human neonatal $\mathrm{Fc}$ receptor show dramatic differences in immunoglobulin $\mathrm{G}$ and albumin binding. J Biol Chem 2010;285:4826-36.

30. Elangbam CS, Qualls CW Jr, Dahlgren RR. Cell adhesion moleculesupdate. Vet Pathol 1997;34:61-73.

31. Volpes R, Van Den Oord JJ, Desmet VJ. Vascular adhesion molecules in acute and chronic liver inflammation. Hepatology 1992;15:269-75.

32. Dillon P, Belchis D, Tracy T, Cilley R, Hafer L, Krummel T. Increased expression of intracellular adhesion molecules in biliary atresia. The Amer J Pathol 1994;145:263-7.

33. Kobayashi H, Horikoshi K, Long L, Yamataka A, Lane GJ, Miyano T. Serum concentration of adhesion molecules in postoperative biliary atresia patients: relationship to disease activity and cirrhosis. J Pediatr Surg 2001;36:1297-301.

34. Patel SJ, Jindal R, King KR, Tilles AW, Yarmush ML. The inflammatory response to double stranded DNA in endothelial cells is mediated by NF?B and TNFa. PLoS ONE 2011;6:e19910.

35. Skurkovich S, Skurkovich B. Anticytokine therapy, especially anti-interferon-gamma, as a pathogenetic treatment in TH-1 autoimmune diseases. Ann N Y Acad Sci 2005;1051:684-700.

36. Mouzaki A, Theodoropoulou M, Gianakopoulos I, Vlaha V, Kyrtsonis MC, Maniatis A. Expression patterns of Th1 and Th2 cytokine genes in childhood idiopathic thrombocytopenic purpura (ITP) at presentation and their modulation by intravenous immunoglobulin G (IVIg) treatment: their role in prognosis. Blood 2002;100:1774-9.

37. Svenson M, Hansen MB, Bendtzen K. Binding of cytokines to pharmaceutically prepared human immunoglobulin. J Clin Investigat 1993:92:253339.

38. Ross C, Svenson M, Nielsen H, Lundsgaard C, Hansen MB, Bendtzen K. Increased in vivo antibody activity against interferon alpha, interleukin1alpha, and interleukin-6 after high-dose Ig therapy. Blood 1997;90:237680.

39. Ross C, Svenson M, Hansen MB, Vejlsgaard GL, Bendtzen K. High avidity IFN-neutralizing antibodies in pharmaceutically prepared human IgG. J Clin Invest 1995;95:1974-8.

40. Ephrem A, Chamat S, Miquel C, et al. Expansion of CD4+CD25+ regulatory $\mathrm{T}$ cells by intravenous immunoglobulin: a critical factor in controlling experimental autoimmune encephalomyelitis. Blood 2008;111:715-22. 